\title{
Ocorrência de Ornithonyssus bursa (Berlese, 1888) (Acari: Macronyssidae) em filhotes de Megascops choliba (corujinha-do-mato) e Pitangus sulphuratus (bem-te-vi), no Rio Grande do Sul, Brasil
}

\author{
Ocurrence of Ornithonyssus bursa (Berlese, 1888) (Acari: Macronyssidae) on Megascops choliba \\ (tropical screech-owl) and Pitangus sulphuratus (great kiskadee) nestlings in the Rio Grande do Sul State, Brazil \\ Carolina S. Mascarenhas ${ }^{1 *}$; Marco A. A. Coimbra²; Gertrud Müller'; João Guilherme W. Brum ${ }^{1}$ \\ ${ }^{1}$ Laboratório de Parasitologia de Animais Silvestres, Instituto de Biologia, Universidade Federal de Pelotas - UFPel \\ ${ }^{2}$ Núcleo de Reabilitação da Fauna Silvestre e Centro de Triagem de Animais Silvestres, Universidade Federal de Pelotas - UFPel \\ Recebido em 19 de Janeiro de 2009 \\ Aceito em 8 de Março de 2009

\section{Resumo} \\ O Núcleo de Reabilitação da Fauna Silvestre e Centro de Triagem de Animais Silvestres da Universidade Federal \\ de Pelotas - RS atendeu dois filhotes de Megascops choliba (corujinha-do-mato) (Strigiformes - Strigidae) e dois de \\ Pitangus sulphuratus (bem-te-vi) (Passeriformes - Tyrannidae) intensamente parasitados por ácaros, em maio de $2005 \mathrm{e}$ \\ dezembro de 2006, respectivamente. Os filhotes e o ninho de P. sulphuratus foram recolhidos na zona urbana da cidade \\ de Pelotas - RS após forte temporal. Os ácaros foram removidos, colocados em álcool $70 \%$ e levados ao laboratório \\ de parasitologia para identificação. Os espécimes foram clarificados em lactofenol, montados em meio de Hoyer e \\ identificados como Ornithonyssus bursa (Acari - Macronyssidae). Registra-se Megascops choliba e Pitangus sulphuratus \\ como hospedeiros de Ornithonyssus bursa, no Rio Grande do Sul, Brasil.
}

Palavras-chave: Ornithonyssus bursa, ectoparasito, corujinha-do-mato, bem-te-vi.

\begin{abstract}
The Center for Rehabilitation of Wildlife and Center for Selection of Wild Animal of the Federal University of Pelotas has attended two nestlings of Megascops choliba (tropical screech-owl) (Strigiformes - Strigidae) and two of Pitangus sulphuratus (great kiskadee) (Passeriformes - Tyrannidae) heavily parasitized by mites, in May 2005 and December 2006, respectively. The nestlings and the nest of P. sulphuratus were collected in the Pelotas urban area after severe storms. The mites were removed, clarified in lactofenol, permanently mounted in Hoyer's medium and identified as Ornithonyssus bursa (Acari - Macronyssidae). Megascops choliba and Pitangus sulphuratus are reported as host of Ornithonyssus bursa in Rio Grande do Sul State, Brazil.
\end{abstract}

Keywords: Ornithonyssus bursa, ectoparasit, tropical screech-owl, great kiskadee.

As aves são hospedeiras de uma rica diversidade de ácaros que infestam penas, pele, vias respiratórias e ninhos. A acarofauna encontrada nos ninhos das aves pode apresentar desde ácaros hematófagos até aqueles que se alimentam de detritos associados ao material dos ninhos. Entre as aves, as espécies hematófagas podem ser vetores de patógenos, como vírus, rickettsias e bactérias (PROCTOR; OWENS, 2000; PROCTOR, 2003).

O Núcleo de Reabilitação da Fauna Silvestre e Centro de Triagem de Animais Silvestres da Universidade Federal de Pelotas

\footnotetext{
*Autor para correspondência: Carolina S. Mascarenhas

Laboratório de Parasitologia de Animais Silvestres, Instituto de Biologia,

Universidade Federal de Pelotas, Campus Universitário, CP 354 ,

CEP 96010-900, Pelotas - RS, Brasil

e-mail: phrybio@hotmail.com
}

(NURFS-CETAS/UFPel) atendeu dois filhotes de Megascops choliba (corujinha-do-mato) e dois de Pitangus sulphuratus (bem-te-vi) em maio de 2005 e dezembro de 2006, respectivamente. No caso de $P$. sulphuratus, os filhotes foram recolhidos juntamente com o ninho. Os quatro filhotes foram encontrados na zona urbana de Pelotas - RS, e transportados em caixas de papeláo pelo Policiamento Ambiental da Brigada Militar ao NURFS/CETAS-UFPel. Foi observada a presença de grande quantidade de ácaros parasitando os filhotes das duas espécies e no ninho de P. sulphuratus. Os artrópodes foram coletados do corpo das aves e do ninho, manualmente, com pinça, conservados em álcool $70 \%$ e encaminhados ao Laboratório de Parasitologia de Animais Silvestres. No laboratório, alguns espécimes foram clarificados em lactofenol, montados permanentemente entre lâmina e lamínula em meio de Hoyer e 
identificados sob microscópio óptico Olympus em aumento de 40X, de acordo com Guimarães, Tucci e Barros-Battesti (2001). O material testemunho foi depositado na Coleção de Artrópodes do Laboratório de Parasitologia de Animais Silvestres.

Os ácaros foram identificados como Ornithonyssus bursa, conhecido como ácaro tropical da galinha ou piolho de galinha. A espécie distribui-se pelas regióes tropicais e subtropicais como parasito de aves domésticas e silvestres. Esse ácaro causa irritaçáo e anemia nas aves, podendo determinar a morte de pintos e até mesmo de aves adultas. Em grandes infestaçóes, as galinhas em choco podem abandonar os ninhos (FLECHTMANN, 1985; GUIMARÁES et al., 2001). No Brasil, não existem dados sobre os efeitos de $O$. bursa em aves silvestres. Em outros países, o impacto do parasitismo por esse ácaro não tem afetado significativamente as aves (POWLESLAND, 1977; STAMP; BRUNTON; WALTER 2002; MØLLER, 2002; BERGGREN, 2005). Os filhotes de corujinhado-mato e bem-te-vi, embora intensamente parasitados, não apresentaram sintomatologia de qualquer enfermidade no momento do exame clínico realizado no NURFS.

Os ácaros foram observados por todo o corpo dos filhotes de ambas as espécies, não sendo identificado um local preferencial, ao contrário do que descreveram Flechtmann (1985) e Guimarães et al. (2001) para pintos, em que $O$. bursa é encontrado preferencialmente alimentando-se ao redor do bico e dos olhos. No entanto, acreditase que as condiçóes em que as aves foram encontradas, além do manuseio e transporte até o NURFS, possam ter influenciado no comportamento dos ácaros.

O encontro de $O$. bursa no ninho de $P$. sulphuratus também foi relato para outras espécies de aves silvestres, como Agelaius xanthomus (yellow-shouldered blackbird) (Passeriformes: Icteridae), em Porto Rico (POST, 1981) e Myiopsitta monachus (caturrita) (Psittaciformes: Psittacidae) na Argentina (ARAMBURÚ; CICCHINO; BUCHER, 2002).

\section{Referências}

ARAMBURÚ, R. M.; CICCHINO, A.; BUCHER, E. Material vegetal fresco en cámaras de cría de la cotorra Argentina (Myiopsitta monachus) (Psittacidae). Ornitologia Neotropical, v. 13, p. 433-436, 2002.

BERGGREN, A. Effect of the blood-sucking mite Ornithonyssus bursa on chick growth and fledging age in the North Island robin. New Zealand Journal of Ecology, v. 29, n. 2, p. 243-250, 2005.

FLECHTMANN, C. H. W. Ácaros de importância médico-veterinária. 3 ed. São Paulo: Nobel, 1985. 192 p.

GUIMARÁES, J. H.; TUCCI, E. C.; BARROS-BATTESTI, D. M. Ectoparasitos de importância veterinária. São Paulo: Plêiade, 2001. $218 \mathrm{p}$.

MØLLER, A. P. Temporal change in mite abundance and its effect on barn swallow reproduction and sexual selection. Journal of Evolutionary Biology, v. 15, p. 495-504, 2002.

POST, W. The prevalence of some ectoparasites, diseases and abnormalities in the yellow-shouldered blackbird. Journal of Field Ornithology, v. 52, n. 1, p. 16-22, 1981.

POWLESLAND, R. G. Effects of the haematophagous mite Ornithonyssus bursa on nestling starling in New Zealand. New Zealand Journal of Zoology, v. 4, p. 85-94, 1977.

PROCTOR, H.; OWENS, I. Mites and birds: diversity, parasitism and coevolution. Trends in Ecology and Evolution, v. 15, n. 9, p. 358-364, 2000.

PROCTOR, H. Feather Mites (Acari: Astigmata): ecology, behavior, and evolution. Annual Review Entomology, v. 48, p. 185-209, 2003.

STAMP, R. K.; BRUNTON, D. H.; WALTER, B. Artificial nest box use by the North Island saddleback: effects of nest box design and mite infestations on nest site selection and reproductive success. New Zealand Journal of Zoology, v. 29, p. 285-292, 2002. 